\title{
THREE DIMENSIONAL CT OF CENTRAL LUNG TUMORS
}

\author{
LESLIE E. QUINT, MD, DANIEL L. MCSHAN, PHD, \\ GARY M. GLAZER, MD ${ }^{1,4}$, MARK B. ORRINGER, $\mathrm{MD}^{3}$, \\ ISAAC R. FRANCIS, MD ${ }^{1}$, AND BARRY H. GROSS, MD ${ }^{1,5}$
}

Three-dimensional computed tomography (CT) reconstructions and conventional CT were compared with surgical/pathological findings in seven patients with central lung tumors, in order to assess accuracy in predicting the lobectomy/pneumonectomy decision. Although conventional CT surpassed 3D CT in diagnosing bronchial tumor invasion, $3 D$ CT appeared to be better for central arterial invasion. Despite problems due to motion effects, partial volume averaging and the time consuming nature of the reconstruction process, $3 D$ CT is probably useful in this setting and promises to have similar applications throughout the body.

KEY WORDS:

Lung neoplasms, CT; Lung neoplasms, surgery;

CT, image display and recording; CT, technology

\section{INTRODUCTION}

The traditional role for computed tomography (CT) in evaluating lung cancer patients has been to stage the primary tumor and assess the thorax and upper abdomen for evidence of local and distant tumor spread. To date, CT has not been used extensively to aid in precise surgical treatment planning of lung

From the Departments of Radiology ${ }^{1}$ [L.E.Q., G.M.G., I.R.F., B.H.G.) and Radiation Oncology ${ }^{2}$, [D.L.M.] and the Department of Surgery, Section of Thoracic Surgery ${ }^{3}$, [M.B.O.] University of Michigan Medical Center, Department of Diagnostic Radiology and Nuclear Medicine, [G.M.G.] Stanford University School of Medicine, and Department of Radiology, [B.H.G.] Henry Ford Hospital.

Address reprint requests to: Leslie E. Quint, M.D., Department of Radiology, Box 0030, University of Michigan Medical Center, 1500 E. Medical Center Drive, Ann Arbor, MI 48109-0030.

Manuscript received April 30. 1990, accepted July 20, 1990.

(c) 1990 by Elsevier Science Publishing Co., Inc.

655 Avenue of the Americas, New York, NY 10010

$0899 / 7071 / 90 / \$ 3.50$ tumors. In a prior study, we evaluated the usefulness of CT in predicting whether lobectomy or pnenumonectomy would be required in patients with central lung masses (1). This prediction is a crucial one in patients with poor pulmonary function who cannot tolerate a pneumonectomy. We found that routine CT had significant limitations and was not of great clinical value in this setting. To determine if this accuracy could be improved by using three-dimensional (3D) reconstructions of CT data, we studied a small set of patients with central lung tumors.

The 3D reconstruction technique used in this study provides a method of producing geometrical surfaces from serial cross-sectional contours. These surfaces can then be graphically displayed and viewed from arbitrary directions. Based on the same CT data which would be studied conventionally, the $3 \mathrm{D}$ reconstruction technique does not add new data to those used in the individual CT slices. Instead, it provides a way to appreciate the three-dimensional shapes of anatomical structures and the spatial relationships between different structures. In this study, we investigated the role of this $3 \mathrm{D}$ CT technique for viability and accuracy in the prediction of lobectomy vs. pneumonectomy.

\section{MATERIALS AND METHODS}

A total of seven patients with central lung tumors was entered into the study group (five retrospectively and two prospectively). All patients in the study group fulfilled the following criteria: 1) presence of central lung tumor 2) good quality intravenous contrast-enhanced thoracic CT scan obtained at our institution for clinical patient management 3) confirmation of the presence or absence of tumor invasion of the pulmonary arteries and the central bronchial tree via intra-operative bronchoscopy, thoracotomy and 
pathology. Conventional scans were obtained using contiguous $10 \mathrm{~mm}$ thick tomographic sections from the lung apices to the level of the caudal liver tip. Each study included dynamic incremental scanning of the pulmonary hila performed during and after an intravenous bolus injection of contrast material (generally $75 \mathrm{cc}$ of Conray $60 \%$ at a rate of $2 \mathrm{cc} / \mathrm{sec}$ ). Scans were obtained using a General Electric 8800 (one patient) or 9800 (six patients) CT/T Scanner (Milwaukee, WI), with scan times of 4.8 or $2.0 \mathrm{sec}$, respectively and interscan delays of 2.5 or $3.5 \mathrm{sec}$, respectively. Images were photographed at standard lung settings (window level $-700 \mathrm{HU}$, width 1000 $\mathrm{HU}$ ) and soft tissue settings (window level $20 \mathrm{HU}$, width $500 \mathrm{HU}$ ).

Hard copy from the axial CT scans was interpreted by two radiologists experienced in chest CT and blinded to both the 3D reconstructions and the surgical findings. If there was a difference of opinion, a consensus was achieved.

Data from the conventional axial CT images were manipulated to generate 3D images using a computerized system developed by members of the Department of Radiation Oncology at our institution. Axial CT images for all patients were video projected onto a large rear projectable digitizer pad and interpreted by a single experienced radiologist who was blinded to the surgical and pathologic data. The projected image size was approximately $50 \times 50 \mathrm{~cm}$. Each identifiable mediastinal and hilar structure (e.g. superior vena cava, aorta, pulmonary arteries, trachea and bronchi, heart, tumor) was manually outlined using an electronic "pencil" and assigned a label and identifying color. High contrast boundaries such as skin and lung margins were outlined automatically by "thresholding," i.e. the region of interest was determined by applying a simple threshold on the CT pixel values. The threshold was selected to be approximately at the midpoint between the average CT pixel values within and without the region of interest. The same threshold level was used for all CT images in the study. With this threshold value, a binary image map was generated for the region of interest on each slice and a simple "left-hand always on the wall" algorithm was used to determine the closed boundary automatically.

The formation of a 3D surface description for each vullined structure was yenerated by approximating each of the contours in the serial set by a uniform (by cord length) mesh of vertices. Vertices on adjacent contours were aligned (or registered) by minimizing the distance betwecn corresponding vertices on adjacent slices. The aligned vertices were then connected by straight line segments resulting in a quadrilateral mesh which was then used to represent the organ surface. A variety of techniques was used to terminate the "top" and "bottom" of each surface, including leaving the surface open, closing the surface, extending the surface, or capping the surface. For the terminating ends of an organ, the surface was "capped" by creating an end point generated by using the centroid of the last contour and extending the point out of that plane to a specified distance usually half of the slice thickness (i.e. at the midpoint between one slice and the next adjacent slice). Bifurcations were handled by adding secondary surfaces; the slice preceding the bifurcation was common to both surfaces.

Each case work-up required approximately 2-5 hours of manipulation by the radiologist. In some cases, an extra hour was required to clean up "glitches" in the contours. The glitches occurred in some of the early cases because of software problems and in a number of the cases because of difficulties in making consistent decisions on contours from slice to slice.

The display of the CT derived anatomical surfaces was accomplished using a VAX 8800 computer and a Gould IP8500 imaging system with a real time digital disk (RTDD). Surface rendering was done completely on the VAX using a gradient shading method with a single infinite light source. Only 16 intensities were used for each surface although a "dithering" technique was used to produce a smoother looking image. The time to render a single structure was slightly under 4 seconds; thus, each complete image required approximately 20 seconds for rendering. To simulate real time rotation, a series of images was generated for a sequential rotation (generally every 5 degrees) and each image frame was stored on the RTDD. Each $3 \mathrm{D}$ reconstruction was generally evaluated using two different rotational axes (generally $\mathrm{X}$ and $\mathrm{Y}$ axes). Replay of the images (at up to 30 frames per second) with user control to stop, advance, and back-up through the rotation sequence was used to evaluate the $3 \mathrm{D}$ presentation of anatomy.

The resulting 3D images were evaluated using a consensus reading by two additional experienced chest radiologists who were blinded to the axial CT data and to surgical data. The radiologist who performed the manual tracings used to generate the 3D reconstructions did not interpret the 3D images.

Conventional axial CT images and 3D CT reconstructions were evaluated for tumor involvement of 1) central pulmonary arteries, i.e. main right or main left pulmonary artery and 2) central bronchi, i.e. main bronchi or both upper and lower lobe bronchi (features which would require pneumonectomy rather than lobectomy for complete tumor resection). Arterial involvement was diagnosed if tumor appeared to 
surround greater than $180^{\circ}$ of the vessel circumference (on the conventional scans all contiguous axial images were taken into account in making this determination). One hundred eighty degrees was arbitrarily chosen as a cut-off measurement in order to maximize both sensitivity and specificity; the $270^{\circ}$ measurement used in our prior study gave very low sensitivity, and we therefore decided to lower this threshold for the current study (1). The diagnosis of bronchial involvement required the presence of abnormal soft tissue within the bronchial involvement required the presence of abnormal soft tissue within the bronchus and/or narrowing of the airway caliber with thickening and irregularity of the walls. Central pulmonary vein involvement was not assessed since the veins were visible on only a limited number of axial sections, making 3D reconstructions of these structures technically difficult.

The presence or absence of tumor invasion of central pulmonary arteries and bronchi was assessed at surgery. Tumor invasion was considered proven when 1) the tumor could not be completely dissected free from the artery or the bronchus, respectively, or 2) when endobronchial tumor was present at bronchoscopy, or 3) when the pathologic specimen showed tumor at the proximal bronchial margin of resection. Findings from the conventional scans and the $3 \mathrm{D}$ reconstructions were compared with each other and with surgical and pathologic findings.

\section{RESULTS}

For the clinical cases studied, four of the seven central lung tumors were in the left upper lobe, two were in the right upper lobe and one was in both the right upper and right lower lobes. There were five squamous cell cancers and two adenocarcinomas. Surgical findings indicated that pneumonectomy was needed to achieve an adequate margin of resection in $5 / 7$ patients due to central arterial involvement only (2 patients) or both central arterial and central bronchial involvement ( 3 patients). In two of these five patients, underlying medical conditions precluded such extensive resections, and more limited resections were performed (left upper lobectomy with left pulmonary arterioplasty in one patient; wedge resection of a right lower lobe mass as well as right apical and posterior segmentectomy for a right upper lobe mass in the other patient). Lobectomy was successfully performed in one patient. The final patient had central bronchial involvement, but underwent successful lobectomy with bronchoplastic sleeve resection.

Evaluation of the axial CT images showed apparent bronchial involvement (either main stem or involving both upper and lower lobe bronchi) in 4 patients. Axial CT interpretations of bronchial involvement were correct in all seven cases (4 true positive, 3 true negative) (Table 1). 3D evaluations suggested bronchial invasion in 3/7 cases; gross tumor invasion was bronchoscopically confirmed in one of these cases (one true positive case: left main bronchus; two false positive cases: one right main bronchus, one right upper lobe and right lower lobe bronchi). In the four cases where bronchial involvement was not suggested on the 3D images, gross bronchial invasion was bronchoscopically proven in two and microscopic invasion was pathologically proven in one (three false negative cases: two left main stem bronchus, one right upper lobe bronchus and bronchus intermedius; one true negative case). Thus, in summary, 3D CT was accurate in predicting central bronchial involvement in 2 cases and inaccurate in five; the inaccuracies occurred in all major bronchi, bilaterally.

On conventional CT images, central arterial invasion was correctly diagnosed in $3 / 7$ cases ( 2 true positive, 1 true negative) and incorrectly diagnosed in $4 /$ 7 cases ( 3 false negative. 1 false positive). Arterial involvement was correctly assessed in $5 / 7$ cases on the 3D reconstructions ( 4 true positives, 1 true negative) and incorrectly assessed in the remaining $2 / 7$ cases ( 1 false negative, 1 false positive).

Axial CT was more accurate $(100 \%)$ in diagnosing bronchial tumor involvement than 3D CT (29\% accuracy) (Table 2) (Figures 1 and 2). The low accuracy of 3D CT reflected both low sensitivity and specificity. Arterial invasion, on the other hand, was better evaluated using 3D CT (71\% accuracy, 80\% sensitivity) than axial CT (43\% accuracy, 40\% sensitivity) (Figure 3).

\section{DISCUSSION}

Results from our previous work (1) indicated that conventional CT was relatively poor in predicting whether lobectomy or pneumonectomy was necessary in order to resect a central lung tumor completely, partly due to low sensitivity in detecting central arterial invasion. In contrast, a study from a different group found that CT was fairly accurate in assessing both central pulmonary arterial and venous invasion (2). However, this report gave few details regarding CT methods, CT diagnostic criteria, or criteria of surgical/pathologic proof. 4/26 cases in our prior study had proven arterial tumor involvement, and CT was only $50 \%$ sensitive in detecting this feature. In the 5 current study cases with proven arterial 
TABLE 1. Correlation of Axial CT and 3D CT Findings with Surgical and Pathologic Proof

\begin{tabular}{|c|c|c|c|c|c|c|c|}
\hline \multirow{2}{*}{$\begin{array}{l}\text { Patient } \\
\text { no. }\end{array}$} & \multirow{2}{*}{$\begin{array}{l}\text { Tumor } \\
\text { location }\end{array}$} & \multicolumn{2}{|c|}{ Axial CT findings@ } & \multicolumn{2}{|c|}{ 3D CT findings@ } & \multirow{2}{*}{$\begin{array}{l}\text { Surgery required } \\
\text { for resection }\end{array}$} & \multirow{2}{*}{$\begin{array}{c}\text { Proven region } \\
\text { of central } \\
\text { bronchial involvement }\end{array}$} \\
\hline & & Artery $^{+}$ & Bronchi* & Artery $^{+}$ & Bronchi* & & \\
\hline 1 & LUL & F- & $\mathrm{T}+$ & $\mathbf{F}-$ & $\mathrm{F}-$ & Pneumonectomy & LMS \\
\hline 2 & LUL & $T+$ & $\mathrm{T}+$ & $\mathrm{T}+$ & $T+$ & Pneumonectomy & LMS \\
\hline 3 & LUL & $\mathbf{F}-$ & $\mathrm{T}+$ & $\mathrm{T}+$ & $\mathrm{F}-$ & Pneumonectomy & LMS \\
\hline 4 & LUL & $\mathrm{F}-$ & $\mathrm{T}-$ & $\mathrm{T}+$ & $\mathrm{T}-$ & Pneumonectomy & - \\
\hline 5 & RUL & $F+$ & $\mathrm{T}-$ & $\mathbf{F}+$ & $\mathrm{F}+$ & Lobectomy & - \\
\hline 6 & RUL & $\mathrm{T}-$ & $\mathrm{T}+$ & $\mathrm{T}-$ & $\mathrm{F}$ & $\begin{array}{l}\text { Lobectomy and } \\
\text { Bronchoplasty }\end{array}$ & RUL \& BI \\
\hline 7 & RUL and RLL & $\mathrm{T}+$ & $\mathrm{T}-$ & $\mathrm{T}+$ & $\mathrm{F}+$ & Pneumonectomy & - \\
\hline
\end{tabular}

$\cdot$ LUL = left upper lobe; RUL = right upper lobe; RLL = right lower lobe

@ $\mathrm{F}=$ false, $\mathrm{T}=$ true

+ involvement of main right or main left pulmonary artery

* involvement of main stem bronchus or both upper and lower lobe bronchi

${ }^{*}$ LMS = left main stem bronchus; RUL = right upper lobe bronchus; BI = bronchus intermedius

involvement, conventional CT was similarly only $40 \%$ sensitive in detecting such invasion, although $3 \mathrm{D} C \mathrm{~T}$ showed increased sensitivity $(80 \%)$. It is possible that both conventional and 3D CT accuracy for arterial involvement could be improved with the use of thinner CT slices. An additional factor contributing to poor CT accuracy in our prior study was the low sensitivity for detection of central bronchial invasion. We postulated that inaccurate evaluation of bronchial involvement might be a particular problem in obliquely oriented bronchi, such as the left main stem bronchus or the left upper lobe bronchus, due to partial volume effects. In the current study, conventional CT was $100 \%$ accurate in diagnosing bronchial invasion, although the sample size was quite small.

The failure of 3D CT to assess bronchial invasion successfully may be related to several factors. Endobronchial or submucosal soft tissue extension are the signs of bronchial invasion seen on conventional CT; both these features are poorly assessed by the surface 3D CT techniques used in this study. Other factors include partial volume averaging (using $10 \mathrm{~mm}$ thick sections) and scan plane misregistration due to varia-

TABLE 2. Accuracy of Axial and 3D CT Findings

\begin{tabular}{|c|c|c|c|c|}
\hline & \multicolumn{2}{|c|}{ Bronchial invasion * } & \multicolumn{2}{|c|}{$\begin{array}{c}\text { Pulmonary artery } \\
\text { invasion }^{+}\end{array}$} \\
\hline & Axial CT & 3D CT & Axial CT & 3D CT \\
\hline Sensitivity & 1.00 & .25 & .40 & .80 \\
\hline Specificity & 1.00 & .33 & .50 & .50 \\
\hline Accuracy & 1.00 & .29 & .43 & .71 \\
\hline Prevalence & .57 & .57 & .71 & .71 \\
\hline
\end{tabular}

* Main stem bronchus or both upper and lower lobe bronchi.

+ Main right or main left pulmonary artery. tions in patient respiration. Partial volume averaging would be reduced using thin CT sections (e.g. 1.5-5 $\mathrm{mm}$ ). However, this would dramatically increase both the number of sections obtained and the amount of time needed to complete a 3D case work up. Although thinner sections would lead to increased susceptibility to scan plane misregistration from minor respiratory variations, this effect would be mitigated in the $3 \mathrm{D}$ reconstruction and presentation process: such small errant excursions would probably appear less important when presented using the more global picture provided by the 3D CT technique.

Three-dimensional reconstructions of conventional axial CT images have been used extensively to study the musculoskeletal system (3-9). However, to our knowledge, there are few published reports on the use of 3D CT for evaluation of soft tissue structures such as the hilum or mediastinum (10). Whereas automatic processing techniques (including thresholding and volumetric rendering) can be used for adjacent structures that differ greatly in density, such as bone and muscle, these automatic techniques are inadequate when applied to adjacent structures of similar densitics, e.g. soft tissues. Thus, until more sophisticated automated edge detection systems are developed, soft tissuc structurc contour definitions must currently be hand-traced.

One major drawback to the use of a manually generated 3D system is the large amount of time (generally several hours) required to set up a typical case presentation. Usually this procedure requires prior training in CT image interpretation, and thus an experienced radiologist is necessary to perform this exercise. In addition, some bias is introduced into the creation of the reconstructions, since the radiologist must use some degree of scan interpretation to create 


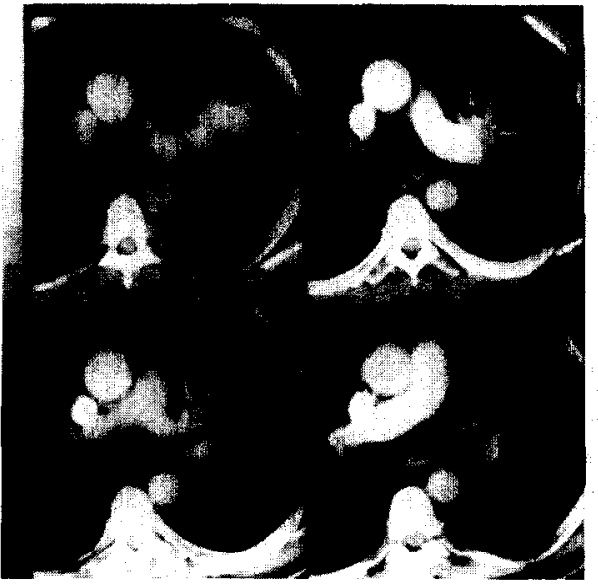

A

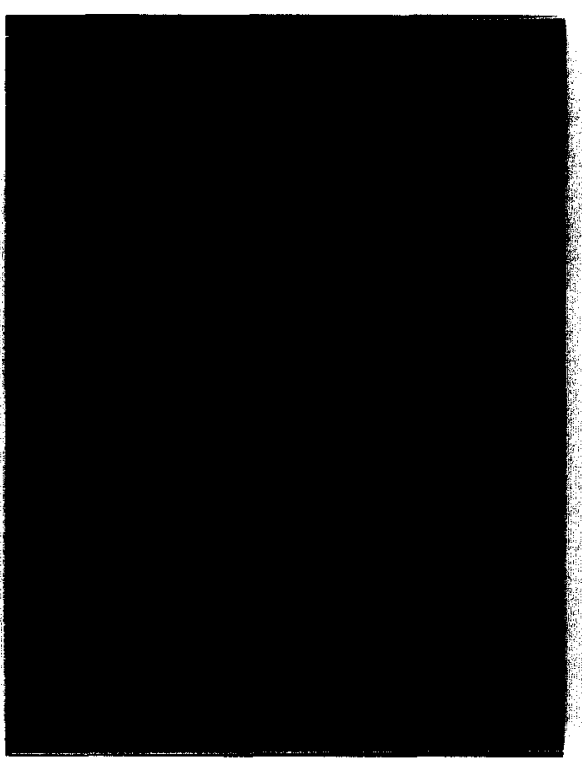

B

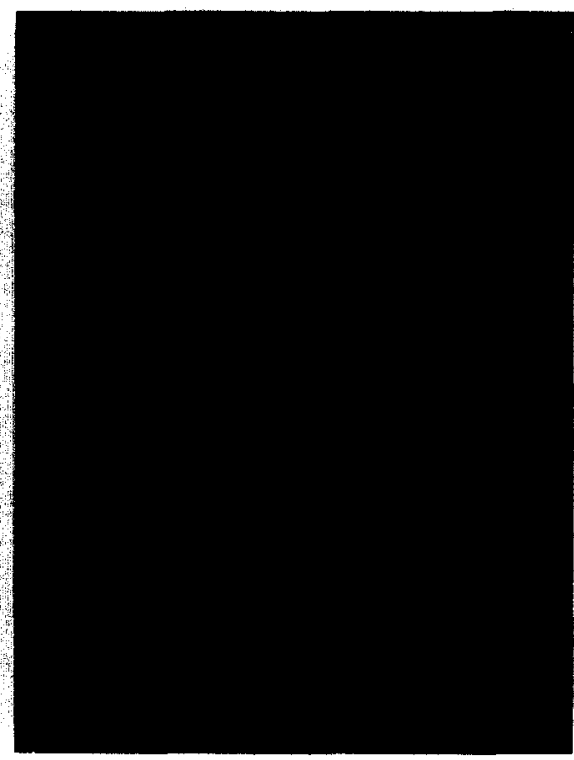

C

Figure 1. Left upper lobe cancer with tumor invasion of left main bronchus and left pulmonary artery (Reprinted, in part, with permission from reference 1). (A) Serial axial C:T scans show tumor (arrows) adjacent to, but not definitely involving, left pulmonary artery. Narrowing of proximal left upper lobe bronchus at its origin suggests tumor extension into left main bronchus (false negative for arterial invasion; true positive for bronchial invasion). (B and C) 3D frontal view (1B) and left lateral view (1C) of heart and hila. Trachea = pink; superior vena cava, right heart and pulmonary arteries $=$ blue; left heart and aorta = red; tumor (arrowheads) = brown. Tumor does not appear to invade either left main bronchus or left pulmonary artery $\left(\leq 180^{\circ}\right.$ contact between tumor and artery). False negative for both arterial and bronchial invasion.

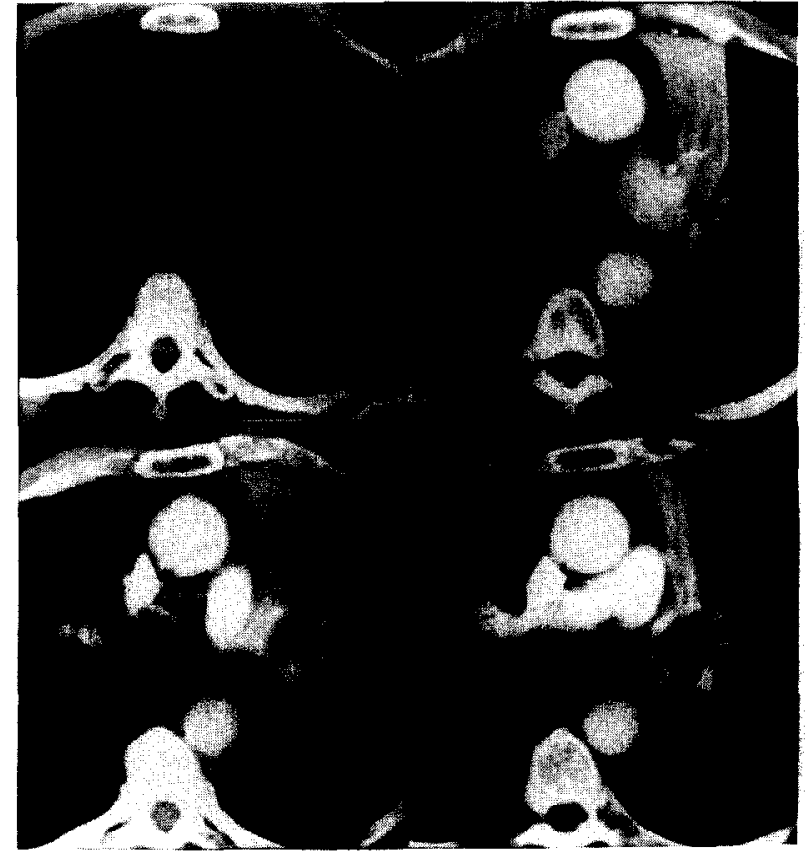

A

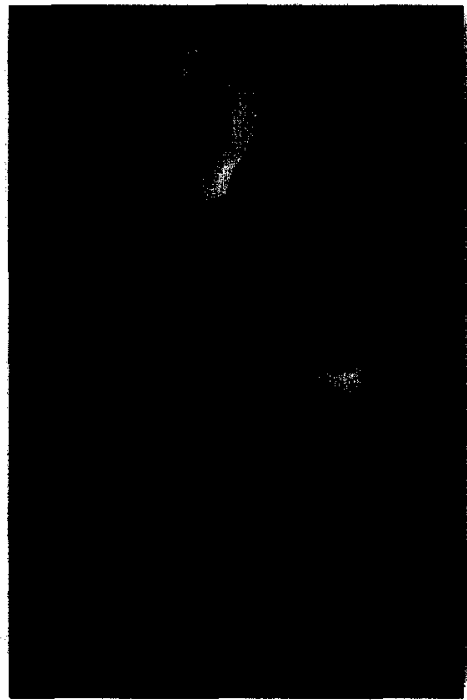

B

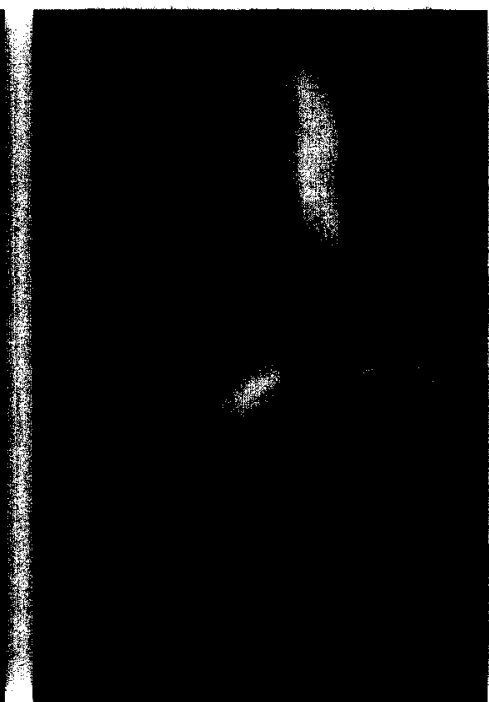

C

Figure 2. Left upper lobe cancer with left pulmonary artery and left main bronchus invasion. (A) Conventional CT false negative for arterial invasion. Tumor (arrowheads) abuts, however does not appear to surround, the left pulmonary artery. True positive for bronchial invasion (arrows). (B and C) 3D reconstruction in LAO (2B) and L.PO (2C) views. True positive for pulmonary artery invasion, false negative for bronchial invasion. Trachea = pink; heart and aorta $=$ red; pulmonary artery = blue; tumor = brown. 
the contour definitions. Even if the contour definitions were assumed to be perfect, the reconstruction and display technique will also affect the case interpretation. A major concern in this study is how well the $3 \mathrm{D}$ reconstruction accurately conveys the shape and relative locations of various organs.

Several types of 3D reconstruction techniques have been reported for CT based image data. The reconstruction technique used for this study is a surface reconstruction algorithim as opposed to a volumetric or voxel type algorithim. Surface renditions are prefered for manually drawn contours represented by polygon line segments. Even though voxel type displays can be generated using the interior of the contoured region, surface renditions produce a more accurate global description of surface curvature. This is because manually entered contours are generally defined by picking only a few points which closely approximate the desired contour when connected with straight lines. In a sense, the manual contouring process serves as a low pass filter preserving the more prominent features. Conversely, if fine structure is important, then surface renditions are inferior to voxel types of displays which (depending on rendering techniques) can demonstrate resolution down to the size of an image pixel.

The accuracy of the surface representation between slices depends on the spacing between slices and on the orientation of the anatomical structure relative to the transaxial CT slices. For example, vessels running parallel to the slices will be difficult to reconstruct, particularly if the diameter is not much bigger than the slice thickness. Volumetric types of displays may appear to provide a better representation in this situation, but would be equally suspect due to partial volume averaging and would be equally dependent on the interpolation scheme used.

Other factors affect the appearance (and subsequent interpretation) of the structures in a generated image. Rendering techniques can strongly affect the appearance of an object. The main factor is the lighting or shading model used. If a constant color was used across the entire surface, and no lighting model applied, then all of the shape detail would be lost inside the projected boundaries as seen from a given viewing direction. On the other hand, some lighting and shading techniques can overemphasize small details. The choice in this study of a uniform infinite source projection tends to be a compromise between the two extremes.

Rendering techniques become less important when the objects portrayed in an image can be rotated interactively. Motion provided one of the strongest three-dimensional cues for this study, with much of the observer insight coming from examination of the "movie-loop" image presentations. Although the images could be played back at 30 frames per second, the most common mode of viewing this data was achieved by slowly stepping through the series of views until a view was obtained which most clearly demonstrated the points of concern in the study. The ability to step the rotation a little beyond and back again was needed to pick the best viewing angle to observe, for example, the maximum (or minimum) separation between two adjacent organs. Often in this study, a selected viewing angle was used to then generate a second (orthogonal) rotation which would provide an even better perspective view. It was frustrating that, for this study, a more interactive viewing technique was not available for true real time rotation of the image from arbitrary angles.

Due to the time-consuming nature of the reconstruction technique, only seven cases were included in this pilot study. Therefore, a rigorous statistical analysis of the data would not be valid. However, analysis of the data suggested that the 3D images were somewhat more helpful in evaluating arterial invasion as compared to the axial images. On the other hand, axial CT appeared to be better than $3 \mathrm{D}$ CT in evaluating the tracheobronchial tree. The improvement in arterial evaluation gained by using $3 \mathrm{D}$ reconstructions was probably due to better $3 \mathrm{D}$ appreciation of relationships between adjacent anatomic structures: despite the fact that radiologists are trained to perform mental 3D reconstructions from 2D data, it is easier even for an experienced crosssectional radiologist to appreciate the full extent of a mass when the image is presented in a 3D format, viewed from any chosen angle while rotating around a desired axis. In addition, we found that the 3D format was very useful in presenting the data to clinical physicians without special training in $2 \mathrm{D}$ image interpretation.

It is clear that methods of automatic edge detection will be necessary in order to make the technique of soft tissue $3 \mathrm{D}$ reconstructions widely applicable in the clinical arena. Such automation will reduce the time required for case processing and will also elininate the bias involved in hand-tracing of structure outlines. Various groups have been working on this problem $(11,12)$, and individuals at our institution arc currently testing and refining edge detection and clustering algorithms to define contours of soft tissue body structures automatically (13). When automation becomes a reality, the $3 \mathrm{D}$ technique can easily be applied to a wide variety of clinical situations, including tumor assessment throughout the body. The current (manual) technique is already extensively 
used at our institution for 3D radiation therapy treatment planning of many tumor types in various body parts. (14-18).

Supported in part by NIH Biomedical Rescarch Support Grant \#RR05383, the General Electric Corporation, and the University of Michigan Cancer Center.

\section{REFERENCES}

1. Quint LE, Glazer GM, Orringer MB. Central lung masses: prediction with CT of need for pneumonectomy versus lobectomy. Radiology 1987; 165:735-738

2. Kameda $\mathrm{K}$, Adachi S, Kono M. Detection of T-Factor in lung cancer using magnetic resonance imaging and computed tomography. J Thorac Imag 1988; 3:73-80

3. Fishman EK, Drebin B, Magid D, Scott WW, Ney DR, Brooker AF, Riley LH, St. Ville JA, Zerhouni EA, Siegelman SS. Volumetric rendering techniques: applications for three-di mensional imaging of the hip. Radiology 1987; 163:737738

4. Fishman EK, Magid D, Ney DR, Drebin RA, Kuhlman JE. Threedimensional imaging and display of musculoskeletal anatomy. J Comput Asst Tomogr 1988; 12:465-467

5. Fishman EK, Drebin RA, Hruban RH, Ney DR, Magid D. Threedimensional reconstruction of the human body. AJR 1988; 150:1419-1420

6. Magid D, Fishman EK, Sponseller PD, Griffin PP. 2D and 3D computed tomography of the pediatric hip. Radiographics 1988; 8:901-933

7. Marsh JL, Vannier MW. Surface imaging from computerized tomographic scans. Surgery 1983; 94:159-165

8. Totty WG, Vannier MW. Complex musculoskeletal anatomy: analysis using three dimensional surface reconstruction. Radiology 1984; 150:173-177
9. Pate D, Resnick D, Andre M, Sartoris DJ, Kursunoglu S. Bielecki D, Dev P, Vassiliadis A. Perspective: three-dimensional imaging of the musculoskeletal system. AJR 1986; $147: 545-551$

10. Herman GT, Liu HK. Display of three-dimensional information in computed tomography. J Comput Assist Tomogr 1977; $1: 155-60$

11. Yang NC, Leichner PK, Fishman EK, Siegelman SS, Frenkel TL, Wallace JR, Loudenslager DM, Hawkins WG, Order SE. CT volumetrics of primary liver cancers. J Comput Assist Tomogr $1986 ; 10: 621-628$

12. Williams DM, Bland P, Liu L, Fargo L. Francis IR, Meyer CR. Liver tumor boundary detection: human observer vs computer edge detection. Investigative Radiology 1989: 24:768-775

13. Fargo L, Williams DM, Bland P, Francis IR, Liu L, Meyer CR. Liver volume determination from CT images with use of histogram cluster analysis. Radiology 1989; 173(P):237

14. Fraass BA, McShan DL. 3.D treatment planning: I. Overview of a clinical planning system. In: Bruinvis I, et al., eds. The Use of Computers in Radiation Therapy. North-Holland: Elsevier, $1987: 273-276$

15. Fraass BA, McShan DL, Diaz RF, et al. Integration of magnetic resonance imaging into radiation therapy treatment plamning. Int J Rad Oncol Biol Phys 1987; 13:1897-1908

16. Lichter AS. Clinical practice of modern radiation therapy treatment planning. In: Paliwal B, Griem M, eds. Syllabus: A Categorical Course in Radiation Therapy: Treatment Planning. Chicago: RSN $\Lambda, 1986: 7-12$

17. McShan DL, Fraass BA. Integration of multi-modality imaging for use in radiation therapy treatment planning. In: Lemke HU, Rhodes ML, Jaffee CC, Felix R, eds. Computer Assisted Radiology. Berlin: Springer-Verlag, 1987:300-304

18. McShan DL, Fraass BA. 3-D treatment planning: II. Integration of gray scale images and solid surface graphics. In: Bruinvis I, et al. eds. The Use of Computers in Radiation Therapy. NorthHolland: Elsevier, 1987:41-44 